\title{
Atom's Nuclear Structure and the Periodic Table of the Chemical Elements
}

\author{
Faustino Menegus \\ V. Europa 15, Bussero, Italy \\ Email: menegus.faustino@gmail.com
}

How to cite this paper: Menegus, F. (2019) Atom's Nuclear Structure and the Periodic Table of the Chemical Elements. World Journal of Nuclear Science and Technology, 9, 140-145.

https://doi.org/10.4236/wjnst.2019.93010

Received: June 9, 2019

Accepted: July 13, 2019

Published: July 16, 2019

Copyright (๑) 2019 by author(s) and Scientific Research Publishing Inc. This work is licensed under the Creative Commons Attribution International License (CC BY 4.0).

http://creativecommons.org/licenses/by/4.0/

\begin{abstract}
A new method for the identification of the chemical Elements isotopes takes advantage of the isotope Neutron Excess (NE) number. The representation of the natural isotopes in the Z-NE plane reveals a surprising correspondence between atom's nuclear and electronic structures. Nuclear directs the atom electronic structure in spite of the alternative set of numbers ruling the two main atom's compartments. These compartments appear better integrated than actually considered. The Mendeleev periodic table is rooted in the atom's nuclear structure. Two recent studies arrive to identical conclusions.
\end{abstract}

\section{Keywords}

Periodic Table, Atom's Structure, Nuclear Structure, Electronic Structure

\section{Introduction}

Atomic nuclei birth takes place in the star interior at temperatures and pressures allowing their nucleosynthesis. Following migration at the star periphery, the reduced temperature and pressure allow nuclei to acquire the necessary electrons to become the stabilized atoms constituting the chemical Elements of our world [1]. The heavy central nuclear, $\mathrm{r}=10^{-15} \mathrm{~m}$ and close to $100 \%$ of the atomic mass, and the external electronic cortex, $\mathrm{r}=10^{-10} \mathrm{~m}$ and close to $100 \%$ of the atomic volume, appear as very distinct and somehow independent atom's compartments. Two distinct branches of physics, nuclear and atomic, describe the above compartments notably ruled by two different sets of numbers: $2,8,20,28$, $50,82,126$ the proton-neutron nuclear magic numbers and $2,10,18,36,54,86$ the noble gases electron numbers, respectively. While the noble gases numbers satisfactory describe the chemical Elements electronic structure and properties, this is not the case for the magic numbers. The nuclear structure is still an open 
field [1]. So different compartments constitute, nevertheless, stable and defined objects: the atoms of the chemical Elements. We suggest here that subtle influences exist between the nuclear and the electronic structure, the latter, likely dictated by the nuclear one. Besides the strong electrostatic interaction between the nucleus and the electrons, a cross-talk of electromagnetic nature takes place between the atom's main compartments: the analysis of atom's electronic spectra allows the retrieval of important nuclear information [2]. Mendeleev maintained that, in addition to the periodic properties, each element has its own personality [3]. Properties like the diagonal behavior, $\mathrm{Li}$ and Be have similarities with $\mathrm{Mg}$ and $\mathrm{Al}$ respectively, or the knight's move, whereby $\mathrm{Zn}$ resembles $\mathrm{Sn}$, may be of nuclear origin. To provide the ground for the discussion of the above ideas, it was decided to employ the representation of the chemical Elements isotopes by the Neutron Excess number [4] [5]. Such a representation contains the basic elements of both the nuclear and electronic atom's compartments.

Recently Mao J., guided by the most abundant isotope of each Element, proposed the atom's nuclear structure as the root of the periodic table [6]. Identical conclusions where reached, Bonchev D. [7], using the tools of the Information Theory [8]. An idea of the present work is contained in a poster presentation at the $15^{\text {th }}$ International Conference on Condensed Matter Nuclear Science: Rome 2009 [9].

\section{Methods}

The Excess Neutron (EN) number of the isotope ${ }_{Z} X^{A}$ of the element $X$ was calculated by the equation: $E N=A-2 Z$. The mass numbers $A$ were from $K$. Lodders [10]. The results constitute a new method for the representation of the chemical Element's isotopes in the Z-NE plane.

\section{Results}

The representation of the chemical Elements isotopes by the Neutron Excess number

In Figure 1, the electronic structure of the Elements is represented by the principal quantum number, $n=1,2,3 \ldots$, and by the orbital angular momentum quantum number, the latter, as usual, by the letters $s, \mathrm{p}, \mathrm{d}$, f. The natural isotopes of the chemical Elements are represented by the atomic $Z$ number and by their Neutron Excess number (NE). With $Z=P=$ proton number, $N=$ neutron number, $A=P+N=$ mass number, $N E=N-P=$ Neutron Excess number, the relationship $A=N E+2 Z$ holds for each isotope represented in Figure 1.

Highlighted in Figure 1 are: the period numbers of the Mendeleev table, the noble gases location and the proton-neutron couples of the nuclear magic numbers. The maximum and minimum $N E$ level of each Element define the borders of the $\beta$-stability valley. The figure inset shows same basic correlations between the isotopes of the chemical Elements. 


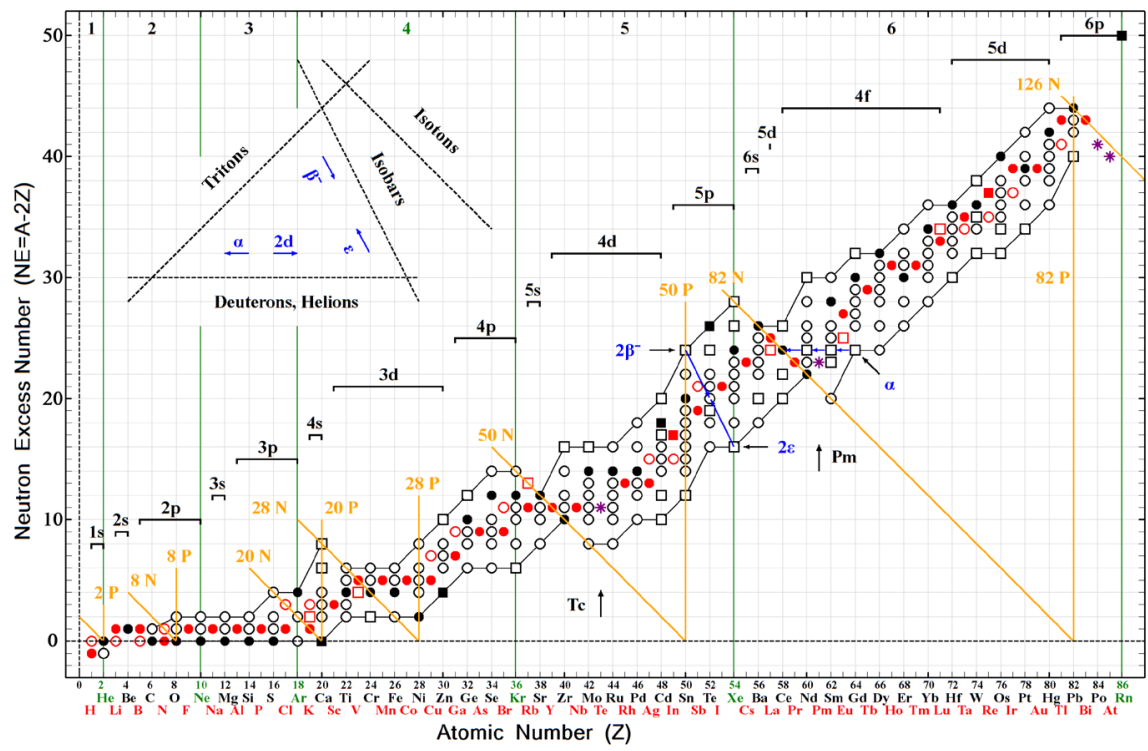

Figure 1. Representation of the Elements isotopes by the Neutron Excess number, $N E=$ $A-2 Z$, in the Z-NE plane and the respective electronic structure. The noble gases limiting the periods of the periodic table, indicated at the top of the figure, are green; s, p, d, f, indicate the electron's angular momentum quantum numbers. Highlighted are the proton-neutron couples of the nuclear magic numbers. Even and odd Element's symbols are black and red respectively; $(O)(\square)$ represent stable and radioactive isotopes respectively, with the full symbol indicating the most abundant isotope of each Element; (*) represents the most stable isotope of the artificial Elements. The inset shows basic relationships between the isotopes along with radioactive decay paths.

What matters to be noted here is that, with the progress of the $Z$ number, the nuclear $E N$ addition takes place with pauses, upsurges and reductions that parallel the periodicity of the chemical Elements properties, the latter marked by the noble gas location. In fact following $\mathrm{He}, \mathrm{Ne}, \mathrm{Ar}, \mathrm{Kr}$ and $\mathrm{Xe}$ there is first a pause, period 2, 3 and 4 or a reduction, period 5 and 6 of the maxima of the NE level addition to the Elements nuclei; see discussion for the anomalous situation at Ca. Most important is the net $N E$ upsurge, coincident with the electron filling of the p orbitals, reaching completeness at the noble gases. The electron filling of the $3 \mathrm{~d}$ orbitals takes place with a clear $E N$ addition pause. That pause, still present in the $4 \mathrm{~d}$ and in the subsequent $\mathrm{f}$ and $\mathrm{d}$ orbitals, progressively vanish because of a robust $N E$ addition. The $N E$ addition is required to counteract the nuclear proton-proton repulsion caused by the $Z$ progress [4]. Closure of the Table periods, with maxima of the nuclear $N E$ levels, is a strong indication of a nuclear periodicity, repeating that of the electronic compartments.

The drop of the $N E$ level behind $\mathrm{Kr}$ and Xe appears directly related to the 50 and 82 isotons. The lack of natural isotopes for Tc and Pm, exactly seven places beyond the noble gases, suggests a gearing of the electronic with the nuclear structure. That these structures are at the least partially geared, is indicated by the identical four proton gap existing between the $50 \mathrm{P}$ and $82 \mathrm{P}$ magic numbers of $\mathrm{Sn}$ and $\mathrm{Pb}$ and the noble gases $\mathrm{Xe}$ and $\mathrm{Rn}$. The most abundant isotope of each 
element convey the same information contained in the upper border of the $\beta$-stability valley, see also [6].

Further support to the idea that the electronic may reflect atom's nuclear structure comes from Figure 2, representing the weighted means of the Elements $N E$ content. Periods 4, 5 and 6 show again a clear correspondence of the electronic with the nuclear structure: a sharp nuclear $N E$ increase accompanies the electron filling of the $\mathrm{p}$ orbitals, reaching maxima at the noble gases. The $N E$ pause after Ar and its reduction after $\mathrm{Kr}$ and Xe mark the electron filling of the $\mathrm{d}$ and $\mathrm{f}$ orbitals. The message of Figure 2 reinforces that of Figure 1 in spite of the notable discrepancy for the light Elements up to Ti. The evolvement of the Elements molar atomic volume [11], with the progress of $Z$, is anything but a monotonous-one, like that of the $N E$ addition to nuclei. The sharp volume increase of the alkaline and alkaline earth metals, coinciding with the $s$ orbitals filling next to the noble gases $N E$ maxima, stresses a nuclear-electronic structure correspondence.

It is well known from inorganic chemistry that in addition to the periodical similarities of $\mathrm{Zn}$ and $\mathrm{Cd}$ with $\mathrm{Cd}$ and $\mathrm{Hg}$ respectively, $\mathrm{Zn}$ and $\mathrm{Cd}$ show in addition similarities with $\mathrm{Sn}$ and $\mathrm{Pb}$ respectively. This last property is known as the Knight's move relationship [12]: one step down and two steps right in the periodic table, as in the game of chess [3]. Figure 2 shows that relationship from the nuclear point of view.

\section{Discussion}

In Figure 1 the ${ }^{46} \mathrm{Ca}$ and the ${ }^{48} \mathrm{Ca}$ isotopes, at variance with the situation in $\mathrm{Mg}$,

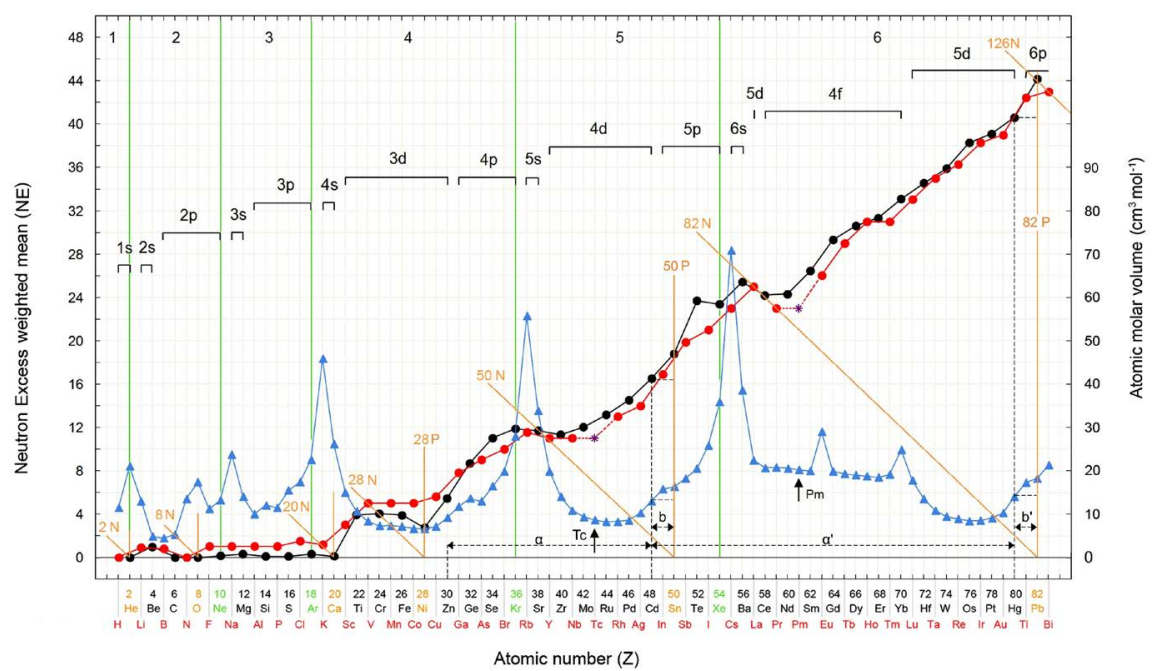

Figure 2. The representation element's $N E$ weighted means and of the Elements Atomic Molar Volumes. The $N E$ means are black or red for even or odd Elements respectively. ( $\Delta$ ) indicate the Atomic molar Volumes. Other symbols as in Figure 1. Both properties show clear periodicity matching that of the Element's electronic structure for periods 4,5 and 6. a, a' and b, b' indicate one step down and two steps right respectively in the periodic table as in the chess Knight's move. 
Sr and $\mathrm{Ba}$, show an abnormal $N E$ increase corresponding to the electron filling of the s orbitals. This exception may be the result of the paramount influence, on the nuclear structure, of the combined effects of the $20 \mathrm{P}$ and the $28 \mathrm{~N}$ magic numbers. The $N E$ drop behind $\mathrm{Kr}$ and $\mathrm{Xe}$, followed by its resumption with the progress of $Z$, is the result of the $N E$ reduction connected to the $50 \mathrm{~N}$ and the 82 $\mathrm{N}$ respectively.

The lack of $N E$ maxima at $\mathrm{Ne}$ and Ar in Figure 2 is the result of the nucleosynthesis mechanisms: nuclear fusion, fading at Fe, for the light Elements, then replaced by the neutron capture processes [1]. The $N E$ drop, connected with the $50 \mathrm{~N}$ and the $82 \mathrm{~N}$ isotons, shows up again with the $126 \mathrm{~N}$ isoton, accompanied by dramatic consequences: beyond $\mathrm{Bi}$ atom's nuclei become unstable. The $N E$ drop is evidenced by the reduced $N E$ content of the most long-lived isotopes of Po and At. A $N E$ recovery takes place with the most long-lived isotope of $\mathrm{Rn}$, confirming $N E$ maxima for the noble gases. Notably the $126 \mathrm{~N}$ isoton does not relate to a noble gas. It relates however with the complete filling of the $4 \mathrm{f}$ and $5 \mathrm{~d}$ orbitals.

At variance with the upper, the lower border of the $\beta$-stability valley doesn't reflect the Element's electronic structure, because it is differently affected by the 50 and 82 neutron magic numbers (Figure 1). As proposed by J. Mao [6], the most abundant isotope of the Elements reflects the atom electronic structure: a suggestion confirmed by the full symbol isotopes in Figure 1. The weighted means of the Elements $N E$ content (Figure 2) confirm again the nuclear-electronic structure correlation.

In Figure 2, the nuclear interpretation of the knight's move shows that moving two steps at the right of $\mathrm{Cd}$ and $\mathrm{Hg}, \mathrm{Sn}$ and $\mathrm{Pb}$ are reached respectively. In both cases the identical nuclear circumstance is found: the shift zeroes the influence of the 50 and 82 neutron magic numbers [4] [5], with the consequent increase of the Elements molar atomic volume [11] and as well as that of the $N E$ level. Changes that may offer a key for the interpretation of the knight's move, presently still debated [13] and, at the same time, may constitute a validation test for the suggestions of the present work.

\section{Conclusion}

The representation of the natural isotopes in the Z-NE plane, together with the basic elements of their nuclear and electronic structures, convey the idea that nuclear directs the atom's electronic structure. In particular, the Element's electronic structure is reflected in the upper border of the $\beta$-stability valley, and in the evolution of the $N E$ number of the most abundant isotope of each Element (Figure 1). This last observation agrees with the findings of J. Mao [6]. Figure 2 beautifully confirms the above conclusions. The Neutron Excess addition mode to nuclei, a determinant of the nuclear stability of the natural isotopes, acts also as a determinant of the atom's nuclear structure. The periodic table of the chemical Elements is rooted in the atom's nuclear structure. 


\section{Acknowledgements}

The author thanks Maurizio Zanardini for the graphical work.

\section{Conflicts of Interest}

The authors declare no conflicts of interest regarding the publication of this paper.

\section{References}

[1] Shaviv, G. (2012) The Synthesis of the Elements. Astrophysics and Space Science Library. Springer, Heidelberg, New York, Dordrecht, London.

https://doi.org/10.1007/978-3-642-28385-7_12

[2] Palffy, A. (2011) Nuclear Effects in Atomic Transitions.

[3] Scerri, E.R. (2007) The Periodic Table. Oxford University Press, Oxford, New York.

[4] Menegus, F. (2016) The Representation of the Chemical Element's Isotopes by the Neutron Excess Content. World Journal of Nuclear Science and Technology, 6, 147-152. https://doi.org/10.4236/wjnst.2016.63016

[5] Menegus, F. (2018) A Suggestion Complementing the Magic Numbers Interpretation of the Nuclear Fission Phenomena. World Journal of Nuclear Science and Technology, 8, 11-22. https://doi.org/10.4236/wjnst.2018.81002

[6] Mao, J. (2017) The Periodic Table Possible Coincided with an Unfolded Shape of Atomic Nuclei. Applied Physics Research, 9, 47-57. https://doi.org/10.5539/apr.v9n6p47

[7] Bonchev, D. (2004) Periodicity of the Chemical Elements and Nuclides: An Information-Theoretical Analysis.

https://www.researchgate.net/..../236671879_Periodicity_of_the

[8] Brillouin, L. (1956) Science and Information Theory. Academic Press, New York.

[9] Menegus, F. (2009) Nuclear and Electronic Structure of Atoms. Proceedings of the 15th International Conference on Condensed Matter Nuclear Science, Rome, 5-9 October 2009, 378-381.

[10] Lodders, K. (2003) Solar System Abundances and Condensation Temperatures of the Elements. The Astrophysical Journal, 591, 1220-1247. https://doi.org/10.1086/375492

[11] Singam, C.N. (1984) Atomic Volume and Allotrpy of the Elements. Journal of Chemical Education, 61, 137-142. https://doi.org/10.1021/ed061p137

[12] Laing, M. (1999) The Knight's Move in the Periodic Table. Education in Chemistry, $36,160-161$.

[13] Rayner-Canham, G. and Oldford, M. (2007) The Chemical Knight's Move Relationship: What Is Its Significance? Foundations of Chemistry, 9, 119-125.

https://doi.org/10.1007/s10698-007-9034-1 